Historic, Archive Document

Do not assume content reflects current scientific knowledge, policies, or practices. 



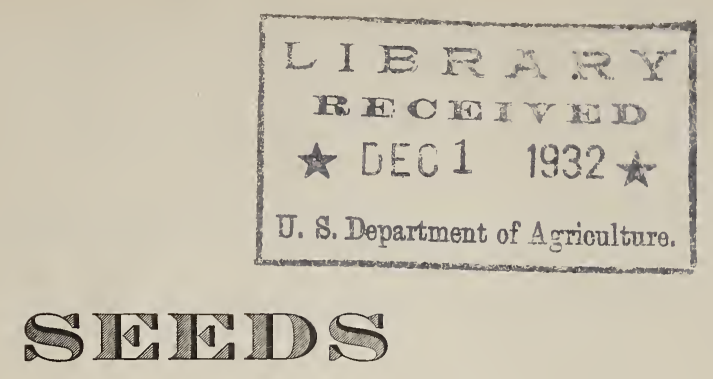

for the

NUTRSTRYMAN ANID HOTRHSTPER

for

Fall and Early Winter

Planting

$1932-1933$

F. W. SCHUMACHER, Horticulturist

Jamaica Plain, Boston, Mass. 
TERMS: as per our General 1932 Catalog.

PAYMENT: CASH, 5\% Discount for Cash with Order.

FOR ADDITIONAL SEEDS NOT LISTED HERE FOR FALL PLANTING CONSULT OUR GENERAL 1932 CATALOG, WHICH LISTS 1200 SEED ITEMS. ALLOWANCES WILL BE MADE ACCORDING TO PROSPECTIVE PRICES FOR 1933.

\section{SEEDS WANTED}

We buy and exchange seeds, also rare and unusual plants which will succeed in our climate.

\section{F. W. SCHUMACHER}

761 Centre Street

Jamaica Plain, Boston, Mass., U. S. A.

Telephone JAMaica 1571

Cable Address - Treeseeds Boston 


\section{CONIFERS: The Most Important Evergreens for Forestry and Ornamental Planting.}

For additional items notlisted in this list, please consult our more complete general catalog of 1932 .

One quarter pound sold at one-third of pound rate; one ounce at one-third of one-quarter pound price.

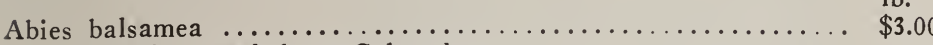

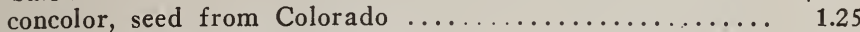

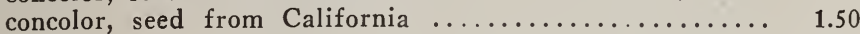

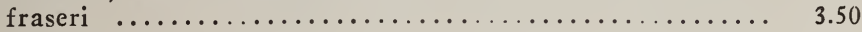

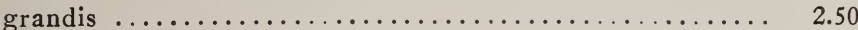

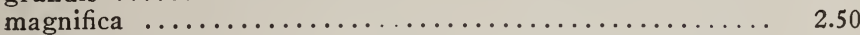

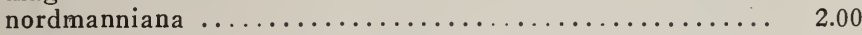

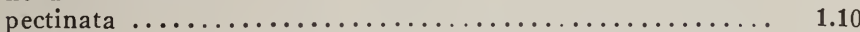

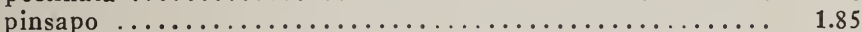

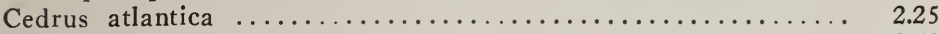

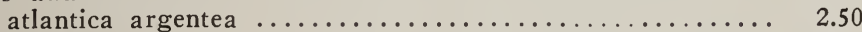

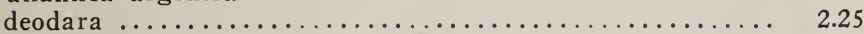

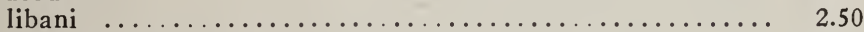

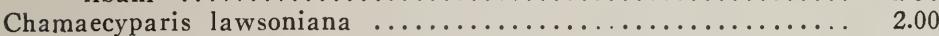

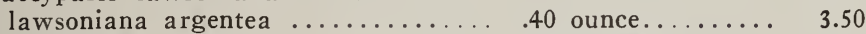

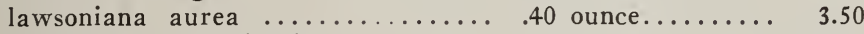

lawsoniana pyramidalis ................ 35 ounce.... 3.00

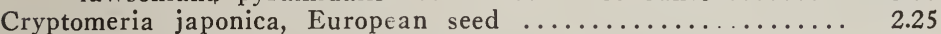

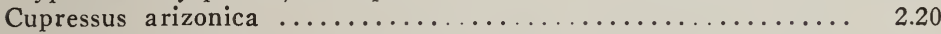

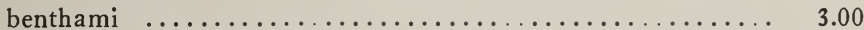

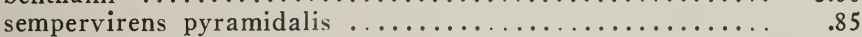

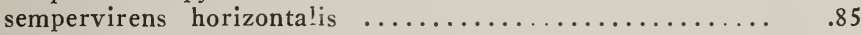

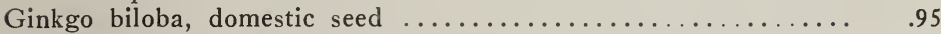

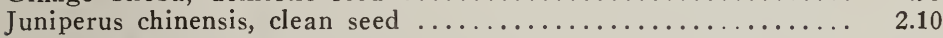

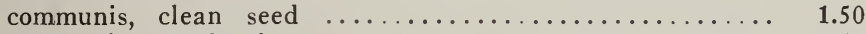

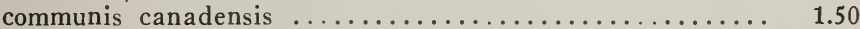

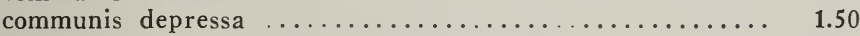

scopulorum, hardy strain from Black Hills ............ 1.60

scopulorum, collected from "Silver Trees" in Montana.... 2.10

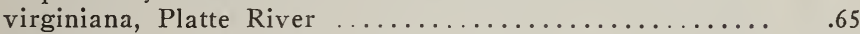

virginiana from cultivated plants, clean seed ..........

Seed from the Alps ...................... 1.50

Large grained strain from western high Alps (6000 feet) . 2.50

europaea sudetica (polonica) the most northerly strain ... 2.25

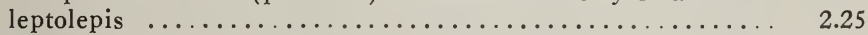

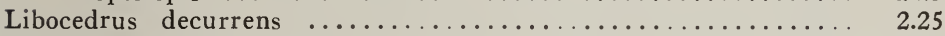

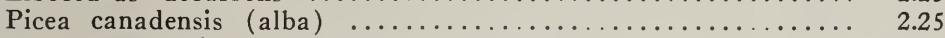

canadensis. Northern New England .............. 4.50

canadensis albertiana, Black Hills Spruce ........... 4.25

engelmanni .................................... 35

excelsa, seed from North Central Europe, $80-85 \%$

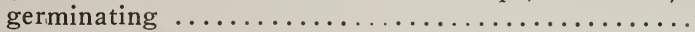
obovata, (Picea excelsa obovata) Siberian Spruce, . 50 ounce $1.501 / 4 \mathrm{lb}$.

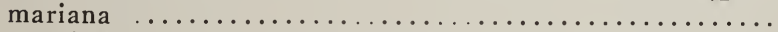
omorika $\ldots \ldots \ldots \ldots .2 .50$ ounce.............

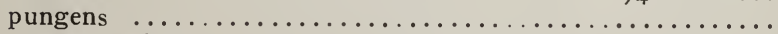

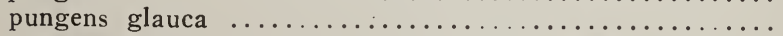
pungens glauca hand picked from Blue trees $\$ 2.501 / 4 \mathrm{lb}$...

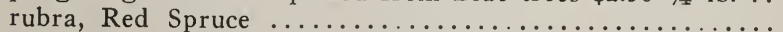
sitchensis, Alaska $6.50 \ldots$ Pacific Coast $\ldots \ldots \ldots \ldots \ldots \ldots \ldots$

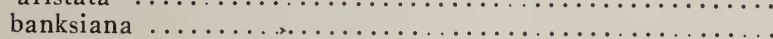

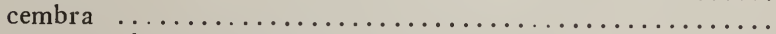

contorta, from Washington $\ldots \ldots \ldots \ldots \ldots \ldots \ldots \ldots \ldots \ldots \ldots \ldots \ldots \ldots \ldots \ldots \ldots \ldots \ldots \ldots \ldots$
contorta, from California $\ldots \ldots \ldots \ldots \ldots \ldots \ldots \ldots$

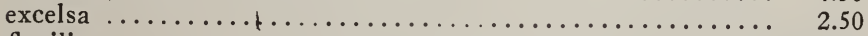

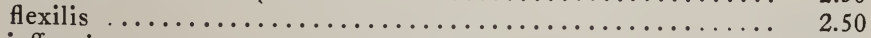

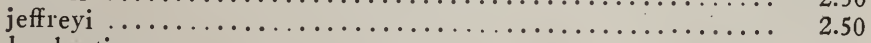

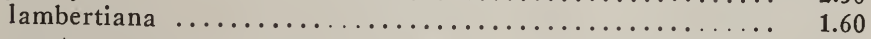

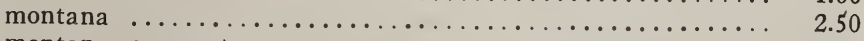

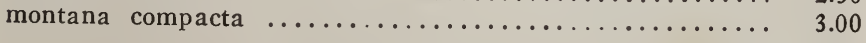




\section{F. W. SCHUMACHER, Jamaica Plain, Boston, Mass.}

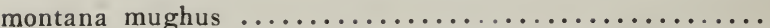
montana mughus selected dwarf strain from Giant Moun-

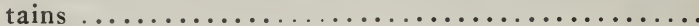

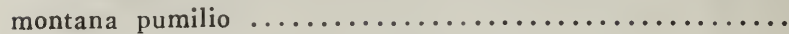

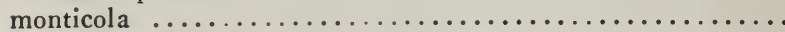
murrayana, inland strain of P. contorta ................

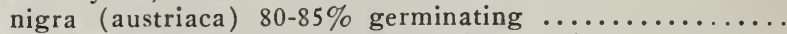
nigra caramanica (Pallasiana) Crimean Pine ..............

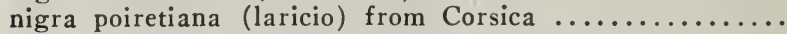

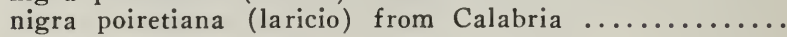

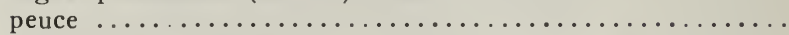

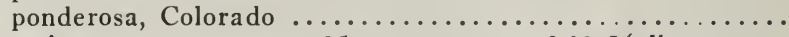
resinosa, ..........95 ounce...... $3.00 \mathrm{r} / 4 \mathrm{lb} . \ldots \ldots$.

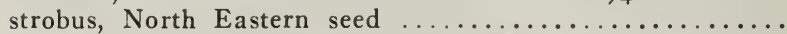
sylvestris, North Eastern Germany, the best Pine seed there

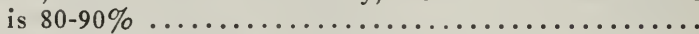
sylvestris from Sweden or Finland $\ldots \ldots \ldots \ldots \ldots \ldots . . . . .$.

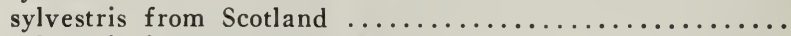

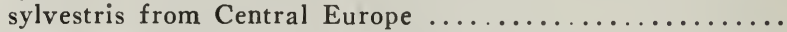

Pseudotsuga douglasi glauca, from Colorado ............. 3.75 douglasi green from Washington $\ldots \ldots \ldots \ldots \ldots \ldots \ldots . . .6 .50$

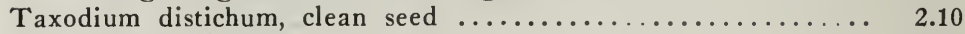

Taxus baccata .............................. 2.00

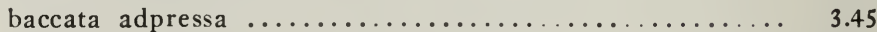

baccata fastigiata ....................... 3.50

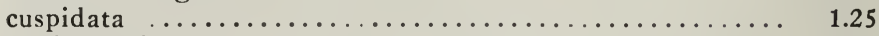

Thuja occidentalis ........................... 2.80

orientalis, domestic seed ...................... 1.50

orientalis aurea ............................ 2.25

orientalis compacta ....................... 2.00

orientalis nana ............................. 2.25

orientalis pyramidalis $\ldots \ldots \ldots \ldots \ldots \ldots \ldots \ldots \ldots \ldots, 2.40$

Tsuga canadensis, Northern New England seed ............. 7.00 caroliniana, here collected from cultivated trees $\$ 1.00$ ounce diversifolia $\ldots \ldots \ldots \ldots \ldots \ldots \ldots \ldots \ldots \ldots \ldots \ldots \ldots, 4.00$

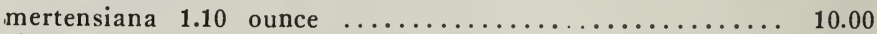

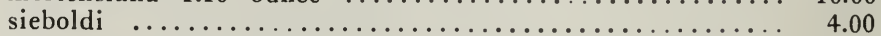

\section{DECIDUOUS SHADE AND FOREST TREES}

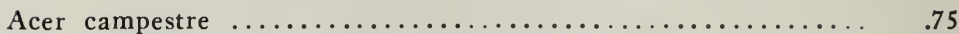

ginnala ................................... 1.50

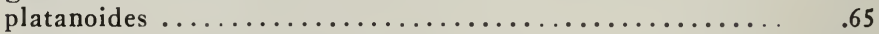

platanoides schwedleri ...................... 1.50

saccharum, Northern New England seed ............ .95

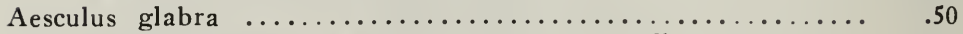

hippocastanum .................... 10 lbs. $1.75 \ldots \ldots \ldots . .25$

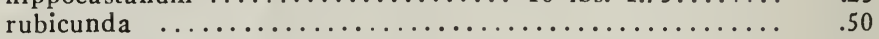

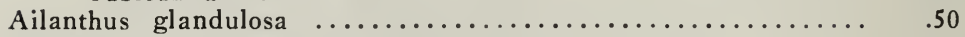

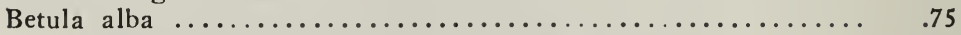

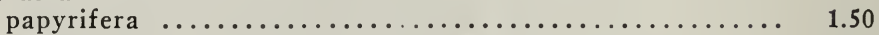

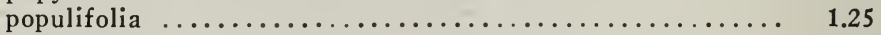

Castanea dentata ................... 10 lbs. $4.00 \ldots \ldots \ldots . .45$

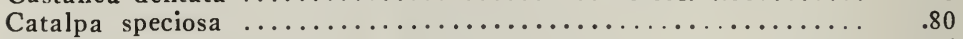

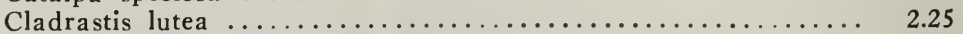

Corylus colurna, Tree Hazel ...................... 1.25

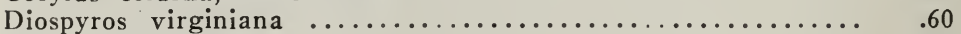

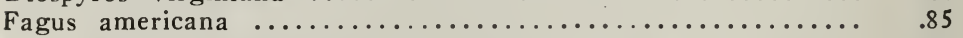

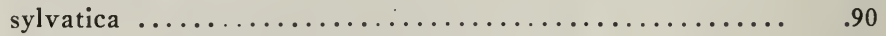

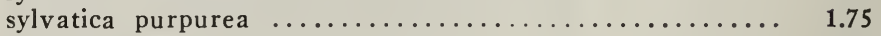

Fraxinus americana $\ldots \ldots \ldots \ldots \ldots \ldots \ldots \ldots \ldots \ldots \ldots \ldots \ldots \ldots \ldots \ldots . . .65$

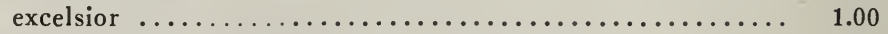

Gleditschia japonica (horrida) ............................. 1.35

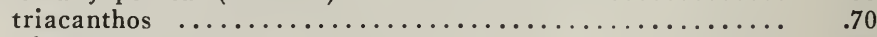

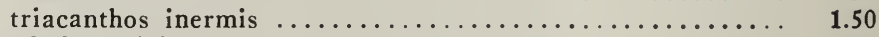

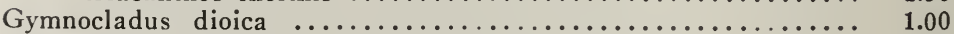

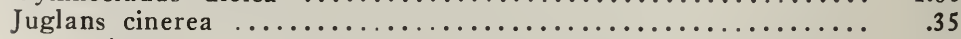

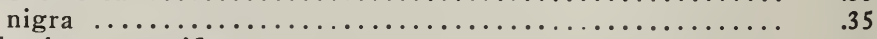

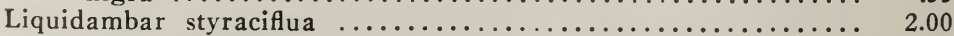

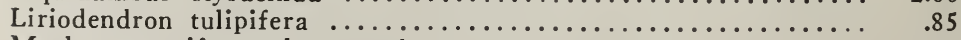

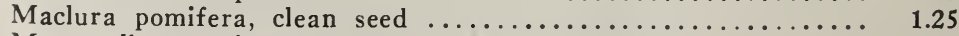

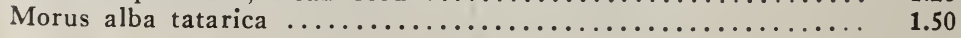




\section{F. W. SCHUMACHER, Jamaica Plain, Boston, Mass.}

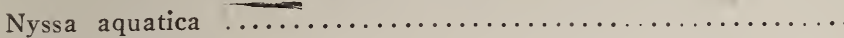

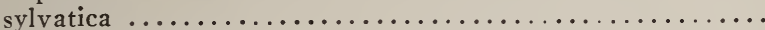

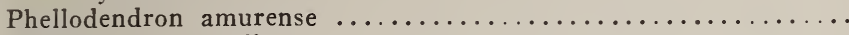

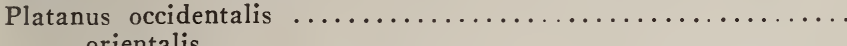

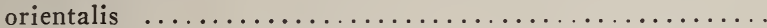

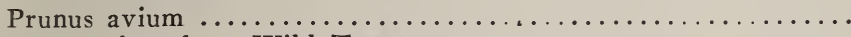

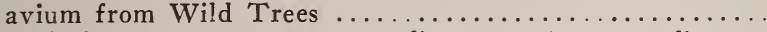

Quercus bicolor .........2.50 $10 \mathrm{lbs} . \ldots \ldots 17.50100 \mathrm{lbs} . \ldots$ coccinea, New England seed, genuine Scarlet Oak

1.7510 lbs......12.00 100 lbs..... macrocarpa, New England seed 2.0010 lbs......16.00 100 lbs.... palustris, New England Seed 2.0010 lbs......16.00 100 lbs..... rubra, New England seed 1.0010 lbs......6. 6.00 100 lbs..... rubra water tested ..1.50 10 lbs..... 8.50100 lbs....

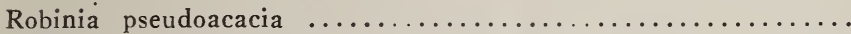

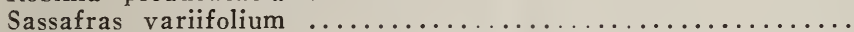

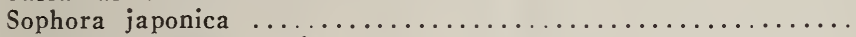

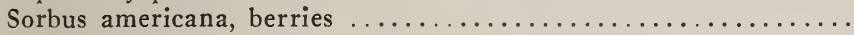

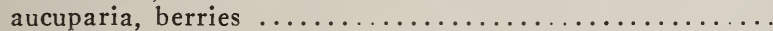

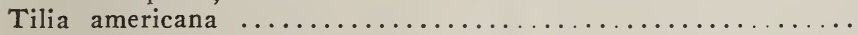

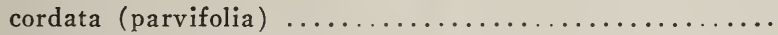

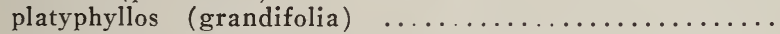

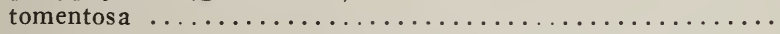

\section{AZALEA AND RHODODENDRON SEEDS}

For More Complete List See Our 1932 GENERAL CATALOG.

Azalea arborescens

1-16 oz. $1 / 4$ oz. oz.

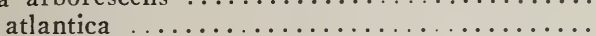
.50

calendulacea, seed from wild plants .......

calendulacea, from plants in cultivation ..

dahurica (Rhododendron dahuricum) ......

dilatata $\quad \ldots \ldots \ldots \ldots \ldots \ldots \ldots \ldots \ldots \ldots \ldots \ldots \ldots \ldots$

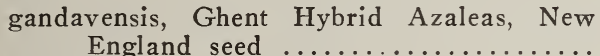

hinodigiri $\ldots \ldots \ldots \ldots \ldots \ldots \ldots \ldots \ldots \ldots \ldots$

indica large flowered from choice collection .................40 pkt....

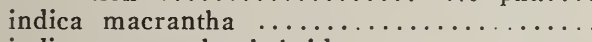

indica macrantha hybrida ......................
japonica, the genuine Japanese Azalea, with flowers of red shades ............ kaempferi, American seed ...... 35 pkt... kaempferi imported ........2.75 r/4 lb....

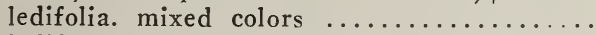
ledifolia alba noordtiana (jap. a. $\operatorname{grd} A$.) ....

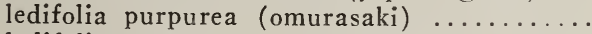
ledifolia rosea $\ldots \ldots \ldots \ldots \ldots \ldots \ldots \ldots \ldots$

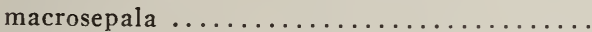
mollis (sinensis) the wild species with yellow flowers mollis, garden strains

seed from a choice New England collec-

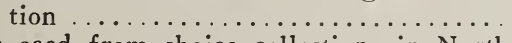

mollis seed from choice collections in North-

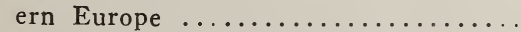

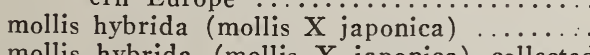
mollis hybrida (mollis $\mathbf{X}$ japonica) collected from "Louisa Hunnewell," 1000 seeds 1.00

mucronulata, (Rhododendron mucron ulatum) seeds from plants in cultivation .75 pkt. mucronulata imported seed ...

$\begin{array}{rrr}.40 & 1.20 & 3.50 \\ 1.25 & 1.70 & 5.00 \\ .20 & .50 & 1.50 \\ .65 & 2.00 & 6.00 \\ .35 & 1.00 & 3.00 \\ .35 & .85 & 2.50\end{array}$
obtusa benigiri ........... .50 $\mathrm{c}$ pkt obtusa kurume, choice mixed colors ......

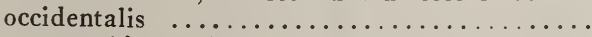
omurasaki, see ledifolia purpurea ......... pontica, true type $\ldots \ldots \ldots \ldots \ldots \ldots \ldots \ldots$

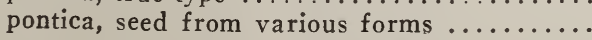


poukhanensis, American seed

$\begin{array}{rrr}.85 & 2.50 & . . \\ . .50 & 1.00 & 3.00 \\ .50 & 1.50 & 4.50 \\ .50 & 1.50 & 4.50 \\ & & . . \\ .70 & 2.00 & 6.00 \\ .25 & .45 & 1.25 \\ . .5 & . .5 & . .25 \\ .20 & .45 & 1.25 \\ .30 & .85 & 2.50\end{array}$

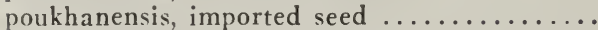

quinquefolia alba $\ldots \ldots \ldots \ldots \ldots \ldots \ldots \ldots$

quinquefolia rosea $\ldots \ldots \ldots \ldots \ldots \ldots \ldots \ldots$

rhombica

rosea, true species of Northern New Eng-
land $\ldots \ldots \ldots \ldots \ldots \ldots \ldots \ldots . .35$ pkt.

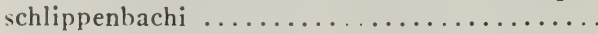

sinensis (see mollis) $\ldots \ldots \ldots \ldots \ldots \ldots \ldots \ldots$

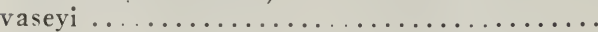

viscosa

2.50

RHODODENDRON: for larger list please consult our 1932 catalog.

ambiguum .

anthopogon

arboreum from cultivated plants

arboreum, red from wild plants

arboreum album, white ........

barbatum

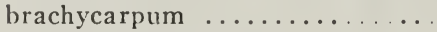

brachycarpum roseum ........

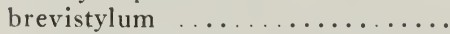

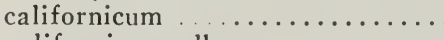

californicum album ..........

camellia eflorum $\ldots \ldots \ldots \ldots \ldots$

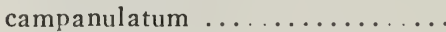

carolinianum, clean seed ........

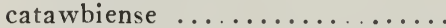

catawbiense compactum ........

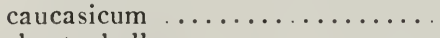

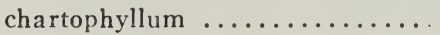

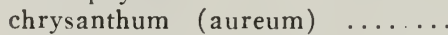

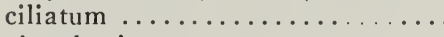

cinnabarinum $\ldots \ldots \ldots \ldots \ldots \ldots$

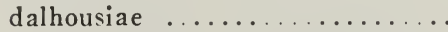

degronianum $\ldots \ldots \ldots \ldots \ldots \ldots$

ferrugineum $\ldots \ldots \ldots \ldots \ldots \ldots \ldots$

fortunei $\ldots \ldots \ldots \ldots \ldots \ldots \ldots$

glaucum $\ldots \ldots \ldots \ldots \ldots \ldots \ldots$

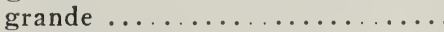

hemitrichotum .............

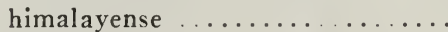

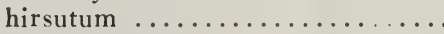

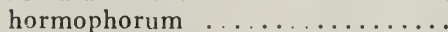

keiskei $\ldots \ldots \ldots \ldots \ldots \ldots \ldots \ldots$

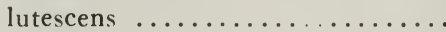

maximum $\ldots \ldots \ldots \ldots \ldots \ldots$

maddeni $\ldots \ldots \ldots \ldots \ldots \ldots \ldots$

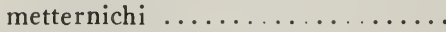

metternichi pentamarum ........

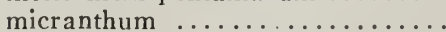

niveum $\ldots \ldots \ldots \ldots \ldots \ldots \ldots$

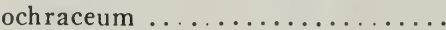

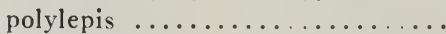

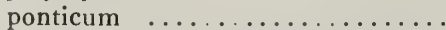

ponticum from hardy specimens in

Northern Europe ..........

pubescens ................

ravum $\ldots \ldots \ldots \ldots \ldots \ldots \ldots \ldots$

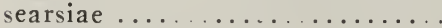

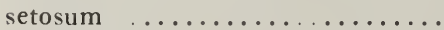

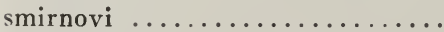

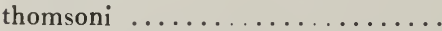

thomsoni candilabrum .........

triflorum ...............

villosum $\ldots \ldots \ldots \ldots \ldots \ldots \ldots \ldots \ldots \ldots$

yanthinum $\ldots \ldots \ldots \ldots \ldots \ldots \ldots$ pkt. 1-16 oz. $1 / 4$ oz. oz. $1 / 4 \mathrm{lb}$.

$.60 \quad 1.75$

$.45 \quad 1.20$

$.15 \quad .25$

.30

.80

.45

.15

.15

.95

.15

.75

.45

.45

.15

.15

.15

.60

.75

.15

.45

.45

.45

.80

.15

.35

.45

.45

.95

.60

.20

.95

.20

.65

.45

..

..

.70

.70

2.00

1.20

1.20
.25

.40

.75

$1.15 \quad 3.50$

..

.45

1.35

1.20

3.60

$1.20 \quad 3.60$

.30

.30

.85

1.00

2.55

.35

1.20

1.20

1.20

30

.30
.75

1.20

1.20

..

1.00

3.00

3.60

..

$\cdots$

.90

2.25

3.00

2.75

..

..

.50

1.50

4.50

.65

2.00

6.00

.30

1.00

3.00

1.20

.20

.. $\quad .25$

.60

1.75

$\begin{array}{ll}.75 & 2.25\end{array}$

$.75 \quad 2.25$

.45

.85

.95

.15

1.20

..

..

.. 


\section{F. W. SCHUMACHER, Jamaica Plain, Boston, Mass.}

\section{HYBRID RHODODENDRONS}

Altaclarense (Arboreum X Ponticum)

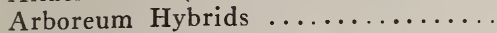

Atrosanguineum, deep blood-red ....

Broughtoni

Catawbiense Hybrids, seed collected from specimen plants in New England

Catawbiense Hybrids, seed from choice hardy varieties in Northern

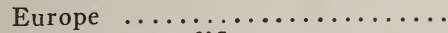

Catawbiense grandiflorum .........

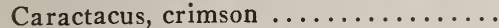

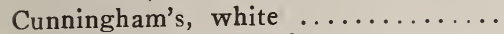

Decorum X Thomsoni ............

Everestianum, rosy-lilac ..........

George Hardy (Auckland Hybrid).

Hybridum (Azaleoides) (Viscosum X

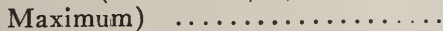

Kettledrum, crimson ............

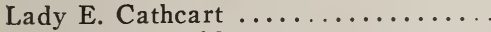

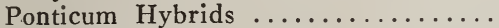

Ponticum X Catawbiense ..........

Silberad's Early Pink ...........

Smirnovi Hybrids ..............

Wellesleyanum (Catawbiense X Maximum) pkt. $1-16$ oz. $1 / 4$ oz. oz. $1 / 4 \mathrm{lb}$. 1.00

$\begin{array}{rrrrr}1.00 & \ldots & \ldots & \ldots & \ldots \\ 1.00 & .35 & 1.00 & 3.00 & \ldots \\ 1.00 & \ldots & \ldots & \ldots & \ldots\end{array}$

$.40 \quad 1.20 \quad 3.50$

$\begin{array}{rrrrr}. & .50 & 1.50 & 4.50 & \ldots \\ .25 & .60 & 1.80 & \ldots & \ldots \\ .35 & 1.00 & \ldots & \ldots & \ldots \\ .95 & \ldots & \ldots & \ldots & \ldots \\ .75 & \ldots & \ldots & \ldots & \ldots \\ .60 & 1.00 & \ldots & \ldots & \ldots \\ 1.00 & \ldots & \ldots & \ldots & \ldots\end{array}$

$\begin{array}{rrrrr}\ldots & .40 & 1.25 & \ldots & \ldots \\ 1.00 & \ldots & \ldots & \ldots & \ldots \\ 1.00 & \ldots & \ldots & \ldots & \ldots \\ .35 & .25 & .75 & 2.00 & \ldots \\ .95 & \ldots 0 & \ldots & \ldots & \ldots \\ .75 & 1.50 & \ldots & \ldots & \ldots \\ & & & \ldots & \ldots\end{array}$

$\begin{array}{lll}.15 & .45 \quad 1.25\end{array}$

\section{SEEDS OF ORNAMENTAL TREES, SHRUBS AND BROADLEAVED ENERGREENS}

A selection of many rare, uncommon or highly recommendable ornamentals offered for fall planting. For more complete list consult our 1932 general catalog which still is in force.

Prices where not stated otherwise are: $1 / 4$ ounce at one-third of ounce rate; $1 / 4$ pound at one-third of pound rate. For larger quantities than indicated request quotations.

AKEBIA lobata

AMELANCHIER canadensis $\ldots \ldots \ldots$

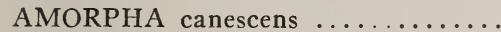

fruticosa $\ldots \ldots \ldots \ldots \ldots \ldots \ldots \ldots$

AMPELOPSIS heterophylla $\ldots \ldots \ldots$

heterophylla amurensis .........

tricuspidata, (veitchi) domestic

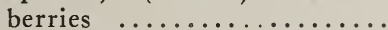

ARALIA spinosa $\ldots \ldots \ldots \ldots \ldots \ldots$

ARONIA arbutifolia ............

melanocarpa $\ldots \ldots \ldots \ldots \ldots \ldots \ldots$

ASIMINA triloba ..............

BERBERIS aggregata, clean seed ..

aggregata pratti .............

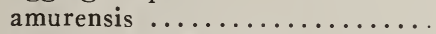

atrocarpa, evergreen .........

brachypoda ...............

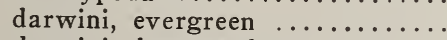

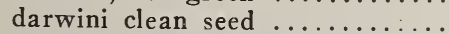

dictophylla, 100 seeds $.50 ; 1000$ seeds $2.50 \quad \ldots \ldots \ldots \ldots \ldots \ldots$.

gagnepaini, evergreen $\ldots \ldots \ldots \ldots$

gagnepaini clean seed..$\ldots \ldots \ldots$

hookeri, evergreen ............

hybrida, Chinese Hybrids ...........

julianae, true to name, 100 seeds $.80 ; 1000$ seeds $\$ 6.00 \ldots \ldots \ldots$

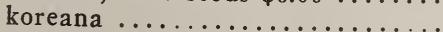

pruinosa $\ldots \ldots \ldots \ldots \ldots \ldots \ldots \ldots \ldots \ldots$

pkt. I/4 oz. oz. I/4 lb. lb.

$\begin{array}{llll}\text {. } & \text {. } & .30 \quad .75\end{array}$

$\begin{array}{lllll}\ldots & \ldots & \ldots & .75 & 2.25\end{array}$

$\begin{array}{lllll}\ldots & \ldots & \ldots & \ldots & 2.25\end{array}$

...$\quad$. $\quad$. $15 \quad .75$

$\begin{array}{lllll}\text {. } & \ldots & .40 & 1.15 & \ldots\end{array}$

$\begin{array}{lllll}. & . & .40 & 1.15 & \end{array}$

$\begin{array}{lllll}\ldots & \ldots & \ldots & \ldots & .85\end{array}$

$\begin{array}{lllll}\ldots & \ldots & \ldots & \ldots & .85 \\ \ldots & \ldots & \ldots & \ldots & 2.00\end{array}$

$\begin{array}{lllll}\ldots & \ldots & \ldots & \ldots & .95\end{array}$

$\begin{array}{lllll}\ldots & \ldots & \ldots & \ldots & .95\end{array}$

$\begin{array}{llllll}\text {. } & \ldots & \ldots & \ldots & 1.25\end{array}$

$\begin{array}{rrrrr}.20 & .30 & .85 & 2.50 & \ldots\end{array}$

$\begin{array}{rrrrr}\ldots & \ldots & .50 & 1.75 & \ldots\end{array}$

$\begin{array}{lllll}\ldots & .35 & 1.00 & 2.75 & 10.00\end{array}$

$\begin{array}{lllll}.50 & \ldots & \ldots & \ldots & \ldots\end{array}$

$\begin{array}{lllll}.30 & \cdots & .60 & 1.75 & .0\end{array}$

$\begin{array}{rrrrr}.35 & .70 & 1.75 & \ldots & \ldots \\ .50 & 1.40 & 4.00 & \ldots & \ldots \\ .50 & \ldots & \ldots & \ldots & \ldots \\ .35 & .60 & 1.80 & \ldots & \ldots\end{array}$

$\begin{array}{rrrr}\ldots & \ldots & \ldots & \ldots \\ \ldots & .40 & 1.15 & 3.50 \\ . & 1.50 & \ldots & \ldots\end{array}$ 
F. W. SCHUMACHER, Jamaica Plain, Boston, Mass.

rubrostilla

.30

sargentiana, true to name .......

pkt. $1 / 4$ oz. oz. $\quad 1 / 4 \mathrm{lb}$.

lb.

sinensis

.50

soulieana, evergreen ........... .20

subcaulialata (Coryi) clean seed

thunbergi, domestic clean seed .. vernae

verrucolosa, evergreen, clean seed verruculosa hybrida, evergreen clean seed .................

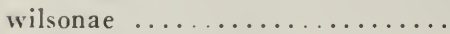

BIGNONIA grandiflora ...........

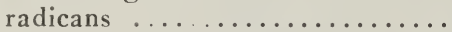

BUXUS microphylla koreana ........

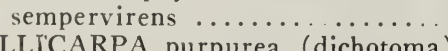

CALLICARPA purpurea (dichotoma)

CALYCANTHUS floridus .......... japonica from European collections

CARAGANA arborescens .........

CARYOPTERIS tangutica ...........

CASSIA marilandica, Indian Senna.

CEANOTHUS americana, clean seed

CELASTRUS scandens .............

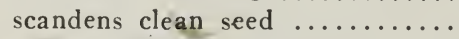

CEPHALANTHUS occidentalis ....

CERCIS canadensis ............... chinensis $\ldots \ldots \ldots \ldots \ldots \ldots \ldots$

CHIONANTHUS virginica $. . . \ldots .$.

CITRUS trifoliata ...............

CLEMATIS coccinea (texensis) .... flammula $. . . \ldots \ldots \ldots \ldots \ldots . . . . .$. koreana (Atrogene koreana) ....

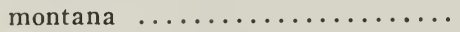
montana grandiflora ............ montana rubens (rosea) ....... paniculata, hand cleaned domestic seed $. . . \ldots \ldots \ldots \ldots . . . . . .$. recta mandshurica ............. tangutica $. . . \ldots \ldots \ldots \ldots \ldots . .$. vitalba $\ldots \ldots \ldots \ldots \ldots \ldots \ldots \ldots$ viticella $\ldots \ldots \ldots \ldots \ldots \ldots \ldots$

COLUTEA arborescens .......... CORNUS alba sibirica, Coral Dogwood, clean seed ........... alternifolia

amomum ...................

florida, Northern seed .........

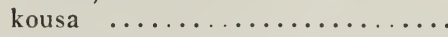

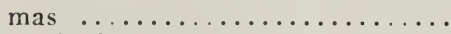

officinalis $\ldots \ldots \ldots \ldots \ldots \ldots \ldots$

paniculata $\ldots \ldots \ldots \ldots \ldots \ldots$.

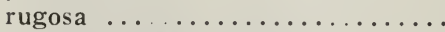

COTONEASTER acuminata .......

acutifolia, clean seed ............ a mbigua................

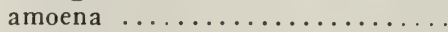
apiculata $\ldots \ldots \ldots \ldots \ldots \ldots \ldots$ applanata, see dielsiana ....... bullata

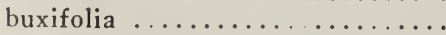
congesta, see microphylla ....... dammeri (humifusa)

dielsiana, clean seed .............

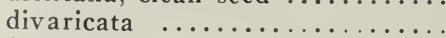
franchetti $\ldots \ldots \ldots \ldots \ldots \ldots \ldots \ldots \ldots$

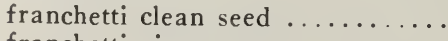
franchetti cinerascens ............

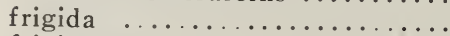
frigida vicari $\ldots \ldots \ldots \ldots \ldots \ldots \ldots$. $\begin{array}{rr}.60 & 1.80 \\ 1.40 & 4.25 \\ \ldots & .50\end{array}$

$\ddot{105}$

$\begin{array}{llll}30 & 8 & 2.0 & . .\end{array}$

$\cdot$ 
F. W. SCHUMACHER, Jamaica Plain, Boston, Mass.

harroviana pkt. $\quad 1 / 4$ oz. oz. $1 / 4 \mathrm{lb}$.

hebephylla

horizontalis, domestic berries ...

horizontalis clean seed ..........

horizontalis perpusilla ...........

humifusa, see dammeri ........

hupehensis, clean seed...$\ldots \ldots$.

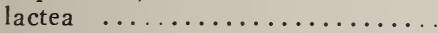

microphylla $\ldots \ldots \ldots \ldots \ldots \ldots$ microphylla clean seed ............ microphylla glacialis (congesta). microphylla thymifolia, clean seed moupinensis .................. moupinensis clean seed ............ nitens $\ldots \ldots \ldots \ldots \ldots \ldots \ldots \ldots \ldots$ pannosa, evergreen ............

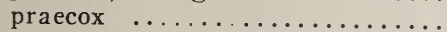
prostrata ..................... racemiflora soongarcia .......... rhytidophylla $\ldots . . . \ldots \ldots \ldots . . . .$. rotundifolia, evergreen ......... salicifolia (salicifolia floccosa) evergreen ................ salicifolia rugosa $\ldots \ldots \ldots \ldots \ldots \ldots$ serotina

simonsi, $\ldots \ldots \ldots \ldots \ldots \ldots \ldots \ldots$

tomentosa, clean seed........ wilsoni

zabeli

CRATAEGUS arnoldiana....... azarolus, clean seed .............. coccinea

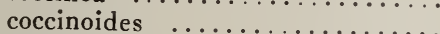
cordata

crusta................... crusgalli $\ldots \ldots \ldots \ldots \ldots \ldots \ldots$. crusgalli clean seed .................. lalandi, clean seed $\ldots \ldots \ldots \ldots \ldots$ macrantha $. . . \ldots \ldots \ldots \ldots . . . . .$. mollis monogyna, clean seed ............. nitida oxyacantha, clean seed rotundifolia persistens ................... punctata $\ldots \ldots \ldots \ldots \ldots \ldots \ldots$ prunifolia $\ldots \ldots \ldots \ldots \ldots \ldots$ succulenta tomentosa multiflorus, White Spanish Broom

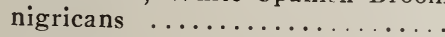
praecox . ................... purgans...$\ldots \ldots \ldots \ldots \ldots$ purpureus, dwarf procumbent ... scoparius

scoparius hardy strain from Scandinavia scoparius Andreana............. scoparius Andreana hybrids .... scoparius sulfureus, Moonlight Broom ..................... supinus (capitatus) $\ldots \ldots \ldots \ldots \ldots$

DAPHNE laureola, clean seed ....... mezereum, clean seed ............

1.25

\section{$.55 \quad 1.60$}

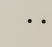




\section{F. W. SCHUMLCHER, Jamaica Plain, Boston, Mass.}

DIERVILLA florida ............... pkt. $1 / 40 z \quad 0 z$

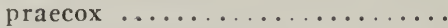

DORYCNIUM hirsutum ......... suff ruticosum

ELEAGNUS angustifolia .........

ELSHOLTZIA stauntoni ...........

EUCALYPTUS globulus .........

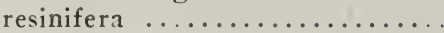

rostrata $\ldots \ldots \ldots \ldots \ldots \ldots \ldots$

$.60 \quad 2.00$

EUONYMUS atropurpureus, clean seed $\ldots \ldots \ldots \ldots \ldots \ldots \ldots$. yedoensis (patens) $\ldots \ldots \ldots \ldots$.

EXOCHORDA grandiflora ..........

GENISTA tinctoria .............

HALESIA diptera ...............

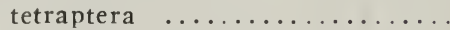

HAMAMELIS japonica arborea .... japonica flavo-purpurascens ..... mollis, we have plants of these, ask for prices

HIBISCUS syriacus .........................

HIPPOPHAE ramnoides, clean seed .

HYDRANGEA arborescens ....... aspera macrophylla ........... heteromalla ................. petiolaris $\ldots \ldots \ldots \ldots \ldots \ldots$. sargentiana $\ldots \ldots \ldots \ldots \ldots \ldots$. xanthoneura wilsoni ...........

HYPERICUM aureum .......... calycinum ................. polyphyllum $\ldots \ldots \ldots \ldots \ldots \ldots$

ILEX aquifolium, clean seed ........ coriacea $\ldots . . \ldots \ldots \ldots \ldots \ldots . . . . .$.

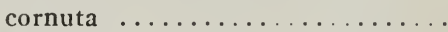
crenata $\ldots . \ldots \ldots \ldots \ldots \ldots \ldots . . .6$.

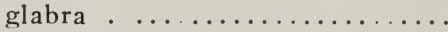
latifolia, berries ............... latifolia clean seed ............. oldhami $\ldots \ldots \ldots \ldots \ldots \ldots \ldots . . . .$. opaca, American Holly, clean Northern seed ........... pedunculosa, clean seed ........ serrata (sieboldi) Japanese Winterberry, clean seed ........ verticillata $\ldots \ldots \ldots \ldots \ldots \ldots$. vomitoria, Yaupon...$\ldots \ldots \ldots$ INDIGOFERA dosua $\ldots \ldots \ldots \ldots$.

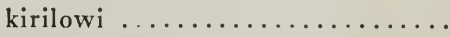

KOELREUTERIA paniculata ...... KOLKWITZIA amabilis, clean seed about 1200 seeds to the ounce LABURNUM alpinum, Scotch Labur-

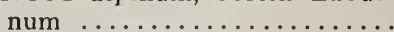
vulgare Goldenchain ............

LAGERSTROEMIA indica ........

LAUROCERASUS caroliniana .....

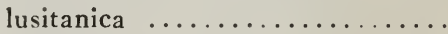

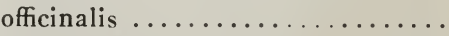

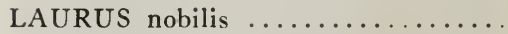

LAVANDULA officinalis (vera) ...

LESPEDEZA bicolor alba ......... bicolor atropurpurea .......... bicolor rosea ..................

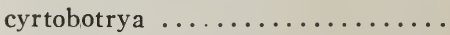
formosa (japonica) ............ formosa albiflora (Desmodium japonicum) 
F. W. SCHUMACHER, Jamaica Plain, Boston, Mass.

MAGNOLIA acuminata $\ldots \ldots \ldots \ldots$ acuminata Northern seed ....... glauca .................... grandiflora, clean seed .......... soulangeana, New. England seed . tripetala $\ldots . . \ldots \ldots \ldots \ldots \ldots$.

MAHONIA aquifolium, clean seed .. aquifolium repens $\ldots \ldots \ldots \ldots$. japonica (bealei) clean seed .... MALUS coronaria, clean seed .......

MYRICA cerifera ................

NANDINA domestica, American seed. berries ..................

PERSEA borbonia Red Bay .......

PHORMIUM tenax .............

PHOTONIA arbutifolia .......... villosa, clean seed ...............

PHYGELIUS capensis coccinea .....

PHYSOCARPUS opulifolius (Spiraea opulifolia) $\ldots \ldots \ldots \ldots \ldots$.

PITTOSPORUM tobira, American seed ....................

POLYGONUM, Fleecevine, auberti .

baldschuanicum ..............

PRUNUS tomentosa ...............

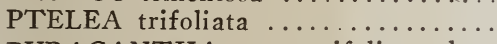

PYRACANTHA angustifolia, clean

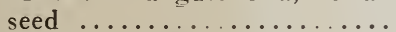
coccinea, clean seed .......... coccinea lalandi ............... coccinea lalandi clean seed .... formosa $\ldots \ldots \ldots \ldots \ldots \ldots \ldots$. yunnanensis, domestic seed ..... yunnanensis clean seed .........

RHAMNUS alaternus, clean seed ... catharticus, clean seed ..........

RHAPHIOLEPIS delacouri ........ indica

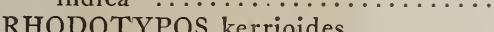

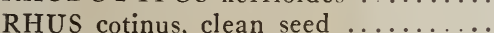
glabra ......................

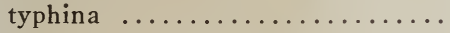

ROSA acicularis nipponensis .......

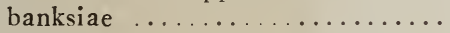

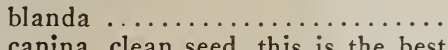
canina, clean seed, this is the best for budding in cold climates and light soil ...10 lbs. 7.50 canina, improved varieties, ask for prices

coreifolia froebeli (laxa), clean

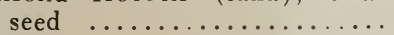

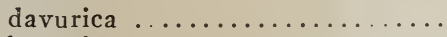

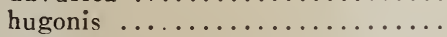

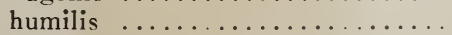

lucida ................... maximowicziana, clean seed ..... moyesi .................... multiflora ........... 10 lbs. 7.00 larger quantities upon request multiflora thornless . .10 lbs. 8.00 larger quantities upon request multiflora nana, Baby Rose 100 hips .... $.50 \quad 1000$ hips 3.50

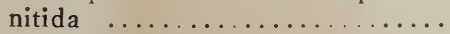
odorata (thea) $\ldots \ldots \ldots \ldots \ldots$. palustris .................... pendulina (alpina)

pkt. $\quad \mathrm{t} / 4 \mathrm{oz} . \quad$ oz. $\quad \mathrm{I} / 4 \mathrm{lb}$.

lb.

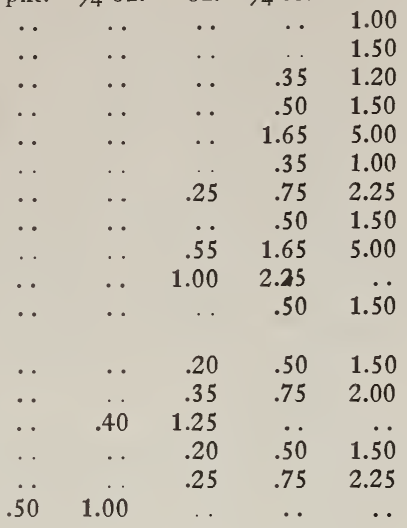

$\begin{array}{llll}. & . & .30 & .80\end{array}$

$\begin{array}{lll}. & . & .60\end{array}$

1.50

.30

.30

....

2.00

2.00

.65

2.00

1.20

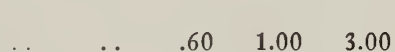

$\begin{array}{rrrrr}. & . . & .60 & 1.00 & 3.00 \\ . & . . & . . & .45 & 1.35\end{array}$

$\begin{array}{lllll}\ldots & . & . & .50 & 1.50\end{array}$

$\begin{array}{llllll}\ldots & \ldots & \ldots & .15 & 2.25\end{array}$

$\begin{array}{lll}.20 & \cdots & .50\end{array}$

.. $\quad . . \quad .20$

. $\quad . . \quad .30$

. $\quad . \quad .25$

.. $\quad$....

. $\quad$. $\quad$.

. $\quad$. $\quad$.

$\begin{array}{lll}\cdots & \cdots & \\ \ldots & \ldots & .20\end{array}$

$\because \cdots$

. $\quad$. $\quad$.

$\begin{array}{lll}. & . & . \\ . & . & .50\end{array}$

....$\quad$.

$\begin{array}{ll}.55 & 1.65\end{array}$

$\begin{array}{ll}.85 & 2.50\end{array}$

.60

$\begin{array}{ll}.60 & 1.75\end{array}$

$.50 \quad 1.50$

$.40 \quad 1.20$

$\begin{array}{ll}.50 & 1.50\end{array}$

$\begin{array}{ll}.60 & 2.40\end{array}$

.. .60

.. $\quad .60$

$\begin{array}{ll}.50 & 1.50\end{array}$

1.50

$.30 \quad 1.00$

.30

.85
2.00

2.20

1.25

$\begin{array}{ll}.40 & 1.25\end{array}$

$\begin{array}{ll}.65 & 2.00\end{array}$

LL 


\section{F. W. SCHUMACHER, Jamaica Plain, Boston, Mass.}

pisocarpa $\because$ (villosa) Apple-Rose pkt. $1-16 \mathrm{oz} .1 / 4 \mathrm{oz} .0 \mathrm{oz} .1 / 4 \mathrm{lb}$. .50

with large fruits fine for preserves, clean seed ..........

rubiginosa, clean seed ........... rubrifolia, clean seed ........... rugosa, clean domestic seed .....

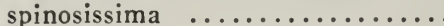

SAMBUCUS nigra, clean seed .... racemosa, clean seed ........... SCHIZOPHRAGMA hydrangeoides.

SKIMMIA japonica .............. SOPHORA vicifolia ..............

SORBARIA (Spiraea) aitchisoni ....

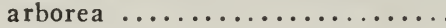
assurgens $\ldots \ldots \ldots \ldots \ldots \ldots \ldots$

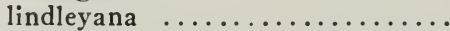

SPARTIUM junceum, Spanish Broom STAPHYLEA pinnata, Bladder-Nut

STYRAX japonica, domestic seed ...

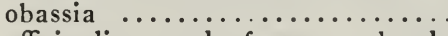
officinalis, seed from a hardy strain from 4000 feet elevation 10 seeds $.35 ; 100$ seeds

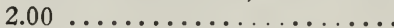

SYRINGA amurensis, clean seed .... dilatata, clean seed........

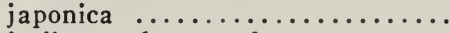
josikaea, clean seed ............ josikaea rosea ...............

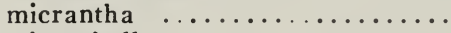
microphylla .................

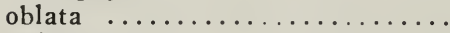
palabiniana ................. tomentella ................. villosa $\ldots \ldots \ldots \ldots \ldots \ldots \ldots$ vulgaris, clean seed ............ vulgaris white flowering, clean seed $\ldots \ldots \ldots \ldots \ldots \ldots$.

vulgaris from double red flowering Hybrids .............. vulgaris from double white flowering Hybrids ................ vulgaris from "Charles $\mathrm{X}$ " ....

americanum, clean seed .........

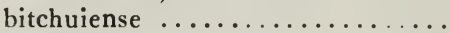

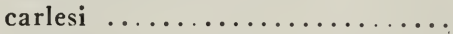
hupehense .................. lantana, clean seed ............. lentago, clean seed ............. lobophvllum .................. odoratissimum, clean seed ......

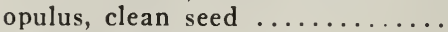
prunifolium ................. rhytidophyllum $. . . \ldots \ldots \ldots \ldots . . .$. theiferum (setigerum aurantiacum) ............... tinus, clean seed ..............

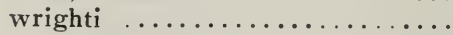

VITEX agnus castus ............. agnus castus, red ............. agnus castus, white ............

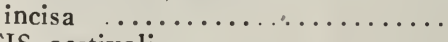

VITIS aestivalis .................. amurensis ....................

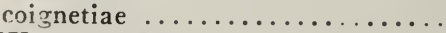


F. W. SCHUMACHER, Jamaica Plain, Boston, Mass.

WISTERIA frutescens $\ldots \ldots \ldots \ldots$. pkt. $1-16$ oz. $1 / 4$ oz. oz. $1 / 4 \mathrm{lb}$.

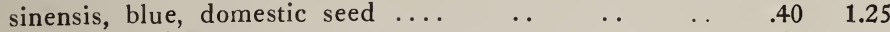
$\begin{array}{llllllr}\text { venusta, white } \ldots \ldots \ldots \ldots \ldots \ldots & \ldots & .30 & .85 & 2.50 & \ldots \\ \text { NTHOCERAS sorbifolia } \ldots \ldots \ldots & \ldots & \ldots & .55 & 1.65 & 5.00\end{array}$

$\begin{array}{llllrrr}\text { XANTHOCERAS sorbifolia } \ldots \ldots \ldots & \ldots & \ldots & .55 & 1.65 & 5.00 \\ \text { YUCCA filamentosa } \ldots . \ldots \ldots \ldots \ldots . & \ldots & \ldots & .25 & .70 & 2.00\end{array}$ gigantea 10 seeds $.40 ; 100$ seeds $2.00 \quad \ldots \ldots \ldots \ldots \ldots \ldots \ldots \ldots \ldots \ldots \ldots \ldots \ldots \ldots \ldots \ldots$ gloriosa $\ldots \ldots \ldots \ldots \ldots \ldots \ldots \ldots \ldots$ hybrida $\ldots \ldots \ldots \ldots \ldots \ldots \ldots \ldots$

PERENNIAL SEEDS FOR FALL AND EARLY WINTER PLANTING

Where not expressly indicated ounce quantities may be obtained at three times the one quarter ounce rate.

Aconitum fischeri $\ldots \ldots \ldots \ldots \ldots \ldots \ldots \ldots \ldots \ldots$

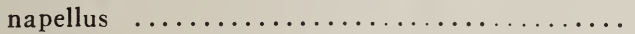

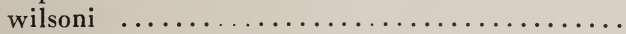

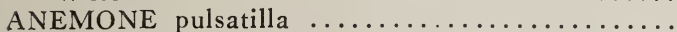

AQUILEGIA alpina $\ldots \ldots \ldots \ldots \ldots \ldots \ldots \ldots \ldots$

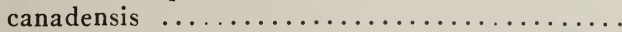
chrysantha grandiflora, large flower, white chrysantha grandiflora sulphurea, large flower,

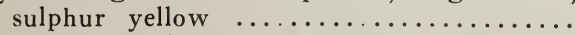
chrysantha new longspurred Hybrids .........

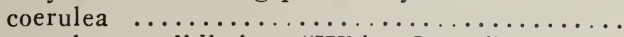
coerulea candidissima "White Queen" .......... coerulea cupreata, "Copper Queen" .........

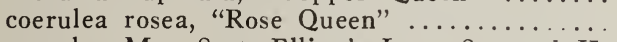
coerulea Mrs. Scott Elliott's Long Spurred Hybrids

New Longspurred Hybrids,

"Copper Queen," a fine novelty with coppery red flowers and dark spur 1.50 $1 / 8$ ounce $\ldots \ldots \ldots \ldots \ldots \ldots \ldots \ldots$

"Emperor," a new giant race, plants 4-5 feet high with fine flowers in good range of colors $.801 / 8$ ounce ........

Pearson's Crimson Star, a long spurred variety which comes true from seed, with white petals and broad sepals of deep blood crimson, the butstanding new Longspurred Columbine, receiving Award of Merit of the Royal Horticultural Society in 1932. 2.25 $\mathrm{r} / 8$ ounce

Pearson's Long spurred pink shades, bright coral pink, $.65 \mathrm{r} / 8$ ounce.............

Pearson's Longspurred Blue Shades of extra good color and texture $.651 / 8 \mathrm{oz}$.

Pearson's Longspurred Lowdham strain, a oxysepala

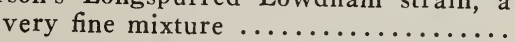

truncata, beautiful dwarf early flowering spe-

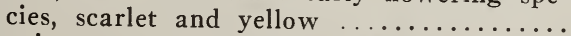
vulgaris compacta erecta "Edelweiss", early flowering new form with upright white flowers, a fine dwarf Columbine for the

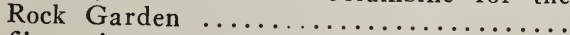
fiine mixture .50 ounce $\ldots \ldots \ldots \ldots \ldots \ldots \ldots \ldots$ nivea grandiflora, fine white, semi-double
ASCLEPIAS TUBEROSA

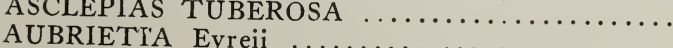
AUBRIETTA Eyreii $\ldots \ldots \ldots \ldots \ldots \ldots \ldots \ldots \ldots$ CAMPANULA persicifolia, fine mixture 1.80 ounce DELPHINIUM, choice Hybrid Strains

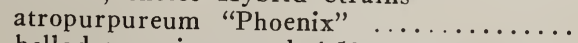
belladonna, improved 4.50 ounce $\ldots \ldots \ldots$. belladona Blue Grotto, fine indigo blue.

\begin{tabular}{|c|c|c|}
\hline \multicolumn{3}{|c|}{ Trade } \\
\hline Pkt. & Pkt. & $1 / 4$ oz. \\
\hline 15 & .35 & .80 \\
\hline .15 & .35 & .50 \\
\hline .20 & .50 & .80 \\
\hline .15 & $\cdots$ & .55 \\
\hline .15 & .35 & .60 \\
\hline .15 & & .40 \\
\hline .15 & .35 & .60 \\
\hline .15 & .35 & .65 \\
\hline .15 & .35 & .60 \\
\hline .20 & .. & .40 \\
\hline .15 & .35 & .70 \\
\hline .45 & 1.00 & 3.00 \\
\hline .15 & .35 & .70 \\
\hline .20 & .40 & .95 \\
\hline
\end{tabular}




\section{F. W. SCHUMUCIER, Jamaica Plain, Boston, IIass.}

pkt. $1-16$ oz. $1 / 4$ oz. oz. $1 / 4 \mathrm{lb}$.

belladona Cliveden Beauty, large flowered, light blue 3.00 ounce ..............

belladonna Fanny Stormouth, very large flowered azure blue 3.00 ounce ......

Continental Hybrids, a development of twenty years of breeding on light soil, in a dry climate with cold winters, selected for clearness of color sturdiness of spikes, mildew resistance. This is perhaps the most thoroughbred race in existence, superior in many ways to English strains 6.00 ounce $\ldots \ldots \ldots$. Named varieties of these:

Albrecht Duerer, light blue with pink; Atlantis, deep azure blue; Blue Jay, light blue and brown; Blue Pot, seagreen, blue; Cathedral-Window, nightblue, late, good crop of second blooms; Castrum Romanum, prussianblue with white, late; Gentian-Tower, pure gentian blue; Gentian Herald, deep blue with black dots, early; Icefountain, shining blue with white; Lilacspear, light violet and blue; Light-tower, iceblue with black, early; Purple Knight, dark blue with gentian blue and white dots; Rokoko, light pink blue; Sailor Boy, dwarf, blue with white; Skyblue, bright sky blue, late; Superman, tall, light blue with white and pink, late; Sunrise, light azure with pink, late; Swedish Blue, blue: Seven Sleepers, light blue, very late flowering; Triumphal Crown, deep dark blue; Watersprite, water blue with mossgreen; Wireless Message, azure, early; Your Blue Miracle, azure, late.

All named varieties as grown by the specialist are 8.50 ounce; $2.85 \mathrm{I} / \mathrm{s}$ ounce; smaller quantities cannot be supplied. ........ elatum, "Iceberg," a white Delphinium which comes true from seed, 4 feet....

Wrexham-Hollyhock flowered Delphinium 5.00 ounce ....................

Wrexham Delphinium, extra select from large flowered double and semidouble named varieties 7.50 ounce ..

EREMURUS bungei, yellow .......1000 seeds 1.75 $.25 \quad .50$

bungei perfectus ............. 1000 seeds 2.50 himalaicus, white ...........1000 seeds 1.50 robustus, pearl color

100 seeds $.60 ; 1000$ seeds 3.50 robustus "Elwesianus" light rose 100 seeds $1.75 ; 1000$ seeds 10.00

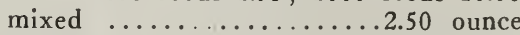

HELLEBORUS niger $\ldots \ldots \ldots \ldots \ldots \ldots \ldots \ldots \ldots \ldots$

IRIS delavayi $\ldots \ldots \ldots \ldots \ldots \ldots \ldots \ldots \ldots \ldots \ldots$

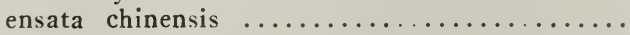
kaempferi ...................75 ounce kaempferi choice double varieties, mixed

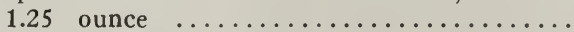
orientalis, "Snow Queen," .........60 ounce

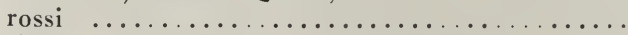
sibirica, "Emperor," a superb Japanese wild form $\ldots \ldots \ldots \ldots \ldots \ldots \ldots \ldots \ldots \ldots$.

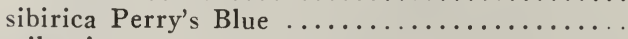
wilsoni $\ldots \ldots \ldots \ldots \ldots \ldots \ldots \ldots \ldots \ldots \ldots \ldots \ldots$ dalmaticum Hybrids 
F. W. SCHUMACHER, Jamaica Plain, Boston, Mass.

Trade

giganteum yunnanense $\ldots \ldots \ldots 1.30 \quad 1-16$ ounce humboldti magnificum ............75 1 1-16 ounce longiflorum praecox, "White Queen,", a fine new white Lily, will bloom within twelve Pkt. Pkt. 1/4 oz. months from sowing $\ldots \ldots .2 .25 \quad 1-16$ ounce

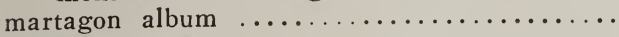
martagon type $\ldots \ldots \ldots \ldots \ldots \ldots \ldots \ldots \ldots \ldots$ philippinense formosanum $\ldots \ldots \ldots \ldots \ldots \ldots$

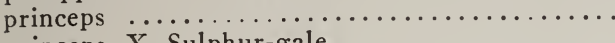
princeps $X$ Sulphur-gale $\ldots \ldots \ldots \ldots \ldots \ldots \ldots$

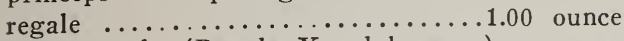
sulphur-gale (Regale $\mathrm{X}$ sulphureum) .......

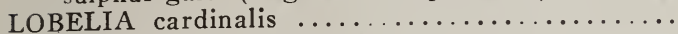
LUPINUS arboreus, Golden yellow ....50 ounce arboreus albus, "Snow Queen" ......60 ounce polyphyllus, the named varieties of Lupins come about $50 \%$ true from seed ............ Blue King, intense blue with very long and elegant spikes ........... 1.50 ounce

Brightest and Best, bright red with long spikes ................50 ounce

Chocolate Soldier, yellow with violet bronze ............2.00 ounce

Cross Roads, beautiful shell pink $1.50 \mathrm{oz}$.

Gold Crest, yellow with deep yellow wings ...............25 ounce

Kingdom, combination of buff with yellow and a very distinct orange flag $2.25 \mathrm{oz}$.

Moerheimi, rose and white, compact habit 1.50 ounce

Perfection, of broad built shape, pure white, in full bloom slightly rose tinged

1.50 ounce

Tangerine, golden yellow with orange terra cotta ..............2.00 ounce

Harkness Regal Hybrids, showing many new and unusual colors ...65 ounce

Queen Hybrids, showing a good percentage of yellows and other rare colors $\$ 1.50$ ounce

Six Hills Sweet Scented Hybrids $1.00 \mathrm{oz}$. MECONOPSIS baileyi .........2.00 1-16 ounce MERTENSIA virginica $\ldots \ldots \ldots \ldots \ldots \ldots \ldots \ldots$

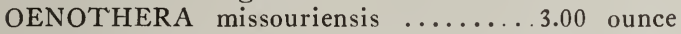

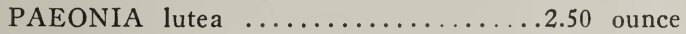
lutea superba $\ldots \ldots \ldots \ldots \ldots \ldots \ldots .75$ ounce

-

. .50 .60

\section{PAPAVER orientale}

Beauty of Livermore, crimson ...2.25 ounce

Mammoth, gigantic dark scarlet flowers, mag-

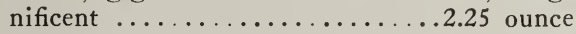

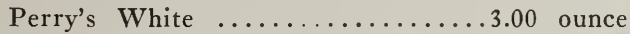
Prince of Orange, scarlet orange ..2.50 ounce Salmon Queen ........................ ounce Mixture of named varieties ........2.00 ounce nudicaule "Baker's Sunbeam" mixture $1.50 \mathrm{oz}$. "Coonara Art Shades" (pink and rose) select stock .............1.80 ounce

"Gibson's New Orange", giant flowered bright growing orange ...2.25 ounce

PENTSTEMON centranthifolius, "Scarlet Bugler", rich vermillion $\ldots \ldots \ldots \ldots \ldots \ldots \ldots \ldots \ldots \ldots$ cobaea, white shades with purple, large flow-

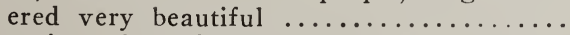
cyananthus, three feet, deep purple blue, early and hardy $\ldots \ldots \ldots \ldots \ldots \ldots \ldots \ldots \ldots \ldots$

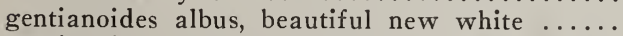
gentianoides Sensation, new select giant flowered ...................... ounce

$\begin{array}{lll}.30 & .50 \quad 1.00\end{array}$




\section{F. W. SCHUMACHer, Jamaica Plain, Boston, Mass. \\ Trade}

gentianoides large flowered, mixed 2.25 ounce gordoni splendens large flowered, brilliant dark

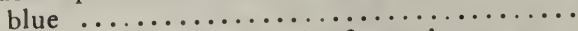

hybridus. "Southgate Gem", fine crimson scarlet ............................... ounce

Pkt. Pkt. 1/4 oz.

$\begin{array}{lll}.15 & .35 & : 75\end{array}$

$\begin{array}{lll}.15 & .25 \quad .60\end{array}$

$.20 \quad .40 \quad 1.00$

isophyllus hybridus, very free and long flowering $2-2 \frac{1}{2} \mathrm{ft}$. tall, flowers on long stalks. beautiful colors, fine for cutting .......... speciosus, deep blue, beautiful .....1.00 ounce

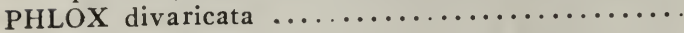

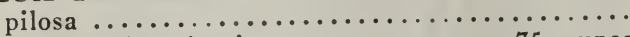

PHYSALIS franchetti .............75 ounce POLEMONIUM coeruleum ............50 ounce reptans $\ldots \ldots \ldots \ldots \ldots \ldots \ldots \ldots \ldots . .00$ ounce PRIMULA auricula gigantea, new giant flowered
$2.50 \mathrm{r} / 8$ ounce beesiana ......................50 ounce

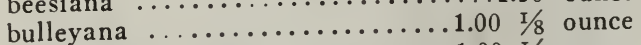
bulleyana hybrida................. $1 / 80$ ounce cashmeriana, new large flowered Hybrids $1.501-64$ ounce denticulata $\ldots \ldots \ldots \ldots \ldots \ldots . .95 \quad 1-32$ ounce denticulata alba $\ldots \ldots \ldots \ldots \ldots . .951-32$ ounce denticulata rosea ...............95 1 1-32 ounce denticulata purpurea ........... 1.25 1-32 ounce denticulata large flowered Hybrids .95 1-32 oz. pulverulenta ................... $80^{\mathrm{r}} \mathrm{8}$ ounce rosea grandiflora ............. 75 1-32 ounce sieboldi ..................2.00 1000 seeds veris elatior, Cowslip alba, pure white .........2.00 $\mathrm{T} / 8$ ounce aurea, golden yellow ........2.20 $\mathrm{r} / 8$ ounce coerulea, blue ..........4.25 1.000 seeds .75100 seeds

finest large flowered mixed (Giant Polyanthus) seed from a specialist $1.50 \mathrm{~T} / 8 \mathrm{oz}$. veris acaulis, dwarf Primrose, charming for Rock Gardens

coerulea, dwarf blue .....3.50 1-32 ounce Virginia, pure white .....2.00 1 -32 ounce new Hybrids ..............1.85 1-32 ounce veitchi .................... $2.00 \quad 1 / 8$ ounce

THALICTRUM dipterocarpum $\ldots \ldots \ldots \ldots \ldots$

THERMOPSIS caroliniana ..........1.35 ounce

TRITOMA hybrida Express, early flowering .... hybrida mirabilis, new Hybrids, all colors .. TROLLIUS caucasicus, Orange Globe $1.25 \mathrm{I} / 8$ ounce europaeus ....................25 ounce new large flowered Hybrids ...............

\begin{tabular}{|c|c|c|}
\hline 25 & .75 & 1.60 \\
\hline .15 & .20 & .35 \\
\hline 20 & .35 & .75 \\
\hline .25 & .50 & \\
\hline . & .15 & .25 \\
\hline & .15 & .20 \\
\hline .20 & .50 & 1.00 \\
\hline .30 & .90 & $\ldots$ \\
\hline .25 & .50 & .. \\
\hline . 20 & .45 & . \\
\hline & .50 & $\ldots$ \\
\hline .. & .30 & .. \\
\hline . & .30 & .. \\
\hline .. & .30 & .. \\
\hline & .30 & .. \\
\hline . & .30 & .. \\
\hline . & .30 & .. \\
\hline & .30 & .. \\
\hline & .40 & .. \\
\hline .35 & .40 & . \\
\hline .35 & .95 & .. \\
\hline .35 & .75 & .. \\
\hline . & .60 & .. \\
\hline & .50 & . \\
\hline & .50 & \\
\hline .25 & .75 & \\
\hline .15 & .35 & .80 \\
\hline .15 & .20 & .40 \\
\hline .15 & .25 & .40 \\
\hline .30 & .60 & 1.30 \\
\hline .25 & .50 & \\
\hline .20 & .45 & 1.00 \\
\hline .30 & .70 & 2.10 \\
\hline
\end{tabular}

